\title{
Forward Modeling of Water-Rich Regions in Mines Based on Unconditional Stability Finite-Difference Time-Domain
}

\author{
Feng Tian", Lingzi Ma and Yuanguo Zhou
}

College of Communication and Information Engineering, Xi'an University of Science and Technology, Xi'an 710054, China

Received 10 September 2018; Accepted 26 January 2019

\begin{abstract}
Water bursting in mines remains one of the major disasters that threatens safe coal production. The traditional detection techniques of water-rich regions in mines can no longer meet new safe production standards and requirements. To solve the constraints on the selection of traditional finite-difference time-domain (FDTD) step length by the CourantFriedrichs-Lewy stability condition, the unconditional stability Crank-Nicolson FDTD (CN-FDTD) algorithm was introduced into the detection of underground total space, aiming to further increase calculation speed and detection accuracy. The boundary condition of the perfectly matched layer (PML) and numerical stability condition for mine detection were analyzed by studying the basic principle of CN-FDTD. Meanwhile, a detection model of underground water-rich regions was constructed. Influences of excitation function, frequency of excitation source, distance between the anomalous body and the interface, and size of the anomalous body on water-rich region detection were analyzed through Wavenology EM and MATLAB simulation by using the forward algorithm. Results demonstrate that given the same conditions, the Ricker wavelet excitation source has better detection effect than the Blackman-Harris Window (BHW) Function, Gaussian and Delta excitation sources. When the anomalous body is $70 \mathrm{~m}$ away from the interface, the excitation source with a central frequency of $15 \mathrm{MHz}$ is the best detection frequency. In addition, forward modeling reveals that when the anomalous body is $50 \mathrm{~m}$ away from the interface, the echo signals of a 3 cubic metres anomalous body could still be detected clearly. Theoretically, the existence of an anomalous body can be determined through this echo signal, and its location can be determined through imaging. The study verifies the effective application of $\mathrm{CN}$ FDTD in water-rich region detection in mines. In addition, CN-FDTD solves the constraints of traditional FDTD by stability condition and increases the accuracy of water-rich region detection in mines. This study provides certain theoretical references for the accurate detection of water-rich anomalous bodies in mines.
\end{abstract}

Keywords: Underground, Total space, Water-rich region, CN-FDTD, Forward modeling, Unconditional stability

\section{Introduction}

Currently, water-bearing fault, water inrush from hidden collapse columns, and water inrush from gob areas are major threats to safe productions in mines. Therefore, the accurate and rapid detection of hidden water-rich regions in mines is important. Currently, the major detection methods that are used to detect hidden water-rich regions in mines are the direct current method, audio scenography, transient electromagnetic method (TEM), and hybrid source electromagnetic method. However, these methods have varying degrees of limitations on detection depth, accuracy, and speed $[1,2]$. To analyze the electromagnetic properties in water-rich regions in mines, a high-efficiency detection method of water-rich regions was improved for threedimensional (3D) forward modeling of total space on the basis of the TEM. This method can recognize the position of the source of water inrush and effectively prevent accidents related to water inrush in mines [3].

On the basis of TEM, the study on one-dimensional (1D) forward and inversion modeling is relatively mature. However, studies on 3D forward and inversion modeling and

*E-mail address: tianfeng0306@163.com

ISSN: 1791-2377 (C) 2019 Eastern Macedonia and Thrace Institute of Technology. All rights reserved. doi:10.25103/jestr.121.24 imaging of underground total space are few. Only few forward and inversion models on 3D time domain are applied to industrial production [4]. A conjugate gradient method for 3D inversion based on TEM was proposed by Haber et al. [4-5]. A 3D forward and inversion modeling was studied by Deng Xiaohong to narrow the spatial range of inversion modeling and shorten its calculation time [4][6]. With an inversion modeling program based on the total space particle swarm optimization developed, the underground TEM in mines was studied by Cheng Jiulong, which effectively increased the accuracy of total space detection [4][7]. The quasi-seismic skewing aperture imaging was investigated by $\mathrm{Li} \mathrm{Xiu}$, which detected the approximate geometric morphology of anomalous bodies [4][8]. The above-mentioned studies are based on traditional finite-difference time-domain (FDTD). The selection of the time step length in FDTD is restricted by stability condition. In other words, the selection of time step length is related to spatial step length. However, the operation efficiency during $3 \mathrm{D}$ total space simulation in underground mines is decreased, thus failing to realize the accurate detection of position and water volume in water-rich regions. Hence, optimizing and improving an unconditionally stable electromagnetic computing method is necessary [3-11].

In this study, the Crank-Nicolson FDTD (CN-FDTD) algorithm is introduced into the underground TEM. An 
underground water-rich region model is constructed to determine the effects of excitation function, frequency of excitation source, distance between the anomalous body and interface, and size of the anomalous body on forward modeling results. The results provide theoretical references for water-rich region detection in mines.

\section{State of the Art}

Several studies on TEM worldwide proved the wide application of TEM in the detection of underground waterrich regions. Based on the original TEM technology, the water-conducting and water-bearing structures with different morphologies at various spatial positions were simulated by Xiuju Xing et al using 3D physical simulation technology, with the fixed-point 3D advanced detection technology designed.. The 3D spatial distribution characteristics of apparent resistivity were analyzed [10]. A groundunderground double source 3D TEM method that combines ground loop emission only and underground overlapping loop emission-acceptance was proposed by Haoli $\mathrm{Xu}$, which increased the detection depth [11]. Using the 3D FDTD method based on Yee grids applied and the traditional Dirichlet boundary conditions replaced by the convolutional perfectly matched layer (CPML), the diffusion law of total space transient electromagnetic field and transient electromagnetic response law in water-rich regions in mines were analyzed by Jianghao Chang [12]. The multiperspective detection for single measuring point was designed by Jingcun $\mathrm{Yu}$ et al. with the "sector scanning" technique of TEM applied, thus enabling to determine the position of water inrush source and direction of water supply on coal face [13]. The water enrichment close to the working face was discussed by Guozhi Tian using complex geophysical prospecting techniques. The anomalous region was delineated according to the contour map of apparent resistivity at the seat floor. Moreover, changes in electric properties along the geophysical prospecting direction were interpreted by combining geographical data. Finally, the water enrichment on the seat floor was delineated [14]. With the single transmitting coil excited and the relative position of receiving coil changed, an underground TEM advanced geology-geophysics detection model was constructed by Guangbo Zhang. The model was used for the physical simulation of typical water-rich regions in mines and was verified by combining the underground test [15]. With the water-rich zoning at the roof of the first working face detected through transient electromagnetic prospecting in mines, the high reliability of transient electromagnetic prospecting and Dupuit's equation on water-rich detection and calculation of water inflow on the roof of the working face were proposed by Wengao Hou et al. [16]. On the basis of summary and analysis of the FDTD algorithm, the unconditional stability Crank-Nicolson $(\mathrm{CN})$ algorithm with high calculation efficiency and high accuracy for anisotropic magnetic plasma medium was proposed by Yongjia Zhuang [17]. The transient electromagnetism numerical simulation method in total space was studied by Zhihai Jiang et al. with the diffusion of the transient electromagnetic field mainly controlled by low specific resistance [18]. With a model of dynamic responses of underground tunnel constructed by Jingcun $\mathrm{Yu}$ et al. using the FDTD method, the horizontal component was proposed as one new method to process and explain dynamic data of mines [19]. The unconditional stability CN-FDTD-PML algorithm that can be solved efficiently was studied and proposed by Haolin Jiang, which increased the calculation efficiency [20-21]. C.T. Schroder et al. investigated the propagation of elastic waves on ground [22]. An algorithm that got solution by the FDTD method on a certain frequency was proposed by Fatih Kaburcuk [23-25]. CN-FDTD was applied to analyze the stability and dispersity of a multi-conductor transmission line by Babak Honarbakhsh et al. [26]. On the basis of the literature review, CN-FDTD has not been applied for 3D electromagnetic surveying in underground total space. Given the low resistance object at one side of the tunnel, the traditional FDTD algorithm and design are influenced by the total space effect. The exciting loop may produce lowresistance exceptional response on the other side of the tunnel. Occasionally, the test might be restricted by tunnel conditions [27-32]. In this study, the traditional FDTD and $\mathrm{CN}$ theory are combined to realize unconditional stability. For the first time, the CN-FDTD electromagnetism algorithm is introduced into mine detection and 3D total space modeling as well as forward modeling on water-rich regions, including water-bearing fault, hidden collapse columns, and water inrush from gob areas.

The remainder of this study is organized as follows. Section 3 shows the basic principle of CN-FDTD and analyzes PML boundaries and numerical stability conditions. Section 4 constructs a water-rich region detection model in mines. Meanwhile, the influences of excitation function, frequency of excitation source, distance between the anomalous body and interface, and size of the anomalous body on forward modeling results are analyzed using a forward modeling algorithm to verify the feasibility of $\mathrm{CN}$ FDTD in water-rich region detection in mines. Section 5 provides the conclusions.

\section{Methodology}

\subsection{Basic principle of CN-FDTD}

The $\mathrm{CN}$ theory is an unconditional stability finite difference method that was proposed by John Crank and Phyllis Nicolson. It is used for the numerical calculation of heat and similar partial differential equations. It is a second-order method on time domain. The $\mathrm{CN}$ theory is hidden in time and can be written as the implicit Runge-Kutta method. Moreover, it is stable in term of numerical value [33, 34].

For the rectangular difference grids in the CN-FDTD algorithm, components $\mathrm{E}$ and $\mathrm{H}$ on any one spatial grid are considered similar to those in the traditional FDTD algorithm. The central difference scheme was used in spatial partial difference, which was also applied to the time partial difference term on the left of the discrete Maxwell equation. However, attention shall be paid to the right term of the equation, which applies the mean of the spatial central difference at $n$ and $n+1$. The CN-FDTD and traditional FDTD algorithms are mainly different in term of time discretization on the right side of the Maxwell equation. [33, 34] A 1D thermodynamic equation was first introduced. The heat conduction equation (or heat equation) is an important partial differential equation. It describes how temperature in one region changes with time:

$$
\frac{\partial u}{\partial t}=c \frac{\partial^{2} u}{\partial x^{2}}
$$

where $u$ is the temperature at one space joint of time and $c$ is a constant parameter related to temperature. 
If the forward difference approximation of the time derivative in the $1 \mathrm{D}$ heat equation is substituted by backward difference and the central difference approximation of spatial derivative is always applied, the heat conduction equation can be rewritten as

$$
\frac{u_{i}^{n+1}-u_{i}^{n}}{\Delta t}=c \frac{u_{i-1}^{n+1}-2 u_{i}^{n+1}+u_{i+1}^{n+1}}{(\Delta x)^{2}}
$$

where $i=1,2,3, \ldots, N-1$ represents space information; and $n=1,2,3, \ldots$ denotes time information.

Then, Eq. (2) can be rewritten as

$$
\frac{u_{i}^{n+1}-u_{i}^{n}}{\Delta t}=\frac{c}{2}\left[\frac{\left(u_{i+1}^{n+1}-2 u_{i}^{n+1}+u_{i-1}^{n+1}\right)}{(\Delta x)^{2}}+\frac{\left(u_{i+1}^{n}-2 u_{i}^{n}+u_{i-1}^{n}\right)}{(\Delta x)^{2}}\right]
$$

This is the $\mathrm{CN}$ equation.

Take the calculation of the electric field intensity $\left(E_{x}\right)$ along the $\mathrm{x}$-axis in the 3D FDTD of the Maxwell equations for example:

$$
\frac{E_{x}^{n+1}-E_{x}^{n}}{d t}=\frac{1}{\varepsilon}\left[\frac{H_{z}^{n+\frac{1}{2}}\left(i+\frac{1}{2}, j+\frac{1}{2}, k\right)-H_{z}^{n+\frac{1}{2}}\left(i+\frac{1}{2}, j-\frac{1}{2}, k\right)}{d y}-\frac{H_{y}^{n+\frac{1}{2}}\left(i+\frac{1}{2}, j, k+\frac{1}{2}\right)-H_{y}^{n+\frac{1}{2}}\left(i+\frac{1}{2}, j, k-\frac{1}{2}\right)}{d z}\right](6)
$$

The forward difference in the form of the next moment in Eq. (6) is replaced by the backward difference. Besides the next moment is

$$
\mathrm{H}^{\mathrm{n}}=\frac{\mathrm{H}^{\mathrm{n}+\frac{1}{2}}+\mathrm{H}^{\mathrm{n}-\frac{1}{2}}}{2},
$$

$$
\frac{E^{n+1}-E_{x}^{n}}{d t}=\frac{1}{\varepsilon}\left[\frac{H_{z}^{n}\left(i+\frac{1}{2}, j+\frac{1}{2}, k\right)-H_{z}^{n}\left(i+\frac{1}{2}, j-\frac{1}{2}, k\right)}{d y}-\frac{H^{n}\left(i+\frac{1}{2}, j, k+\frac{1}{2}\right)-H_{y}^{n}\left(i+\frac{1}{2}, j, k-\frac{1}{2}\right)}{d z}\right]
$$

The backward difference of Eq. (7) at the next moment is replaced by the forward difference, obtaining the following:

$$
\frac{E_{x}^{n+1}-E_{x}^{n}}{d t}=\frac{1}{\varepsilon}\left[\frac{H^{n+1}\left(i+\frac{1}{2}, j+\frac{1}{2}, k\right)-H_{z}^{n+1}\left(i+\frac{1}{2}, j-\frac{1}{2}, k\right)}{d y}-\frac{H_{y}^{n+1}\left(i+\frac{1}{2}, j, k+\frac{1}{2}\right)-H_{y}^{n+1}\left(i+\frac{1}{2}, j, k-\frac{1}{2}\right)}{d z}\right]
$$

Finally, Eqs. (7) and (8) are combined, and the central difference is selected:

$$
\begin{aligned}
& \frac{E_{x}^{n+1}-E_{x}^{n}}{d t}=\frac{1}{\varepsilon}\left[\frac{H_{z}^{n+1}\left(i+\frac{1}{2}, j+\frac{1}{2}, k\right)+H_{z}^{n}\left(i+\frac{1}{2}, j+\frac{1}{2}, k\right)-H_{z}^{n+1}\left(i+\frac{1}{2}, j-\frac{1}{2}, k\right)+H_{z}^{n}\left(i+\frac{1}{2}, j-\frac{1}{2}, k\right)}{2 d y}\right. \\
& \left.-\frac{H_{y}^{n+1}\left(i+\frac{1}{2}, j, k+\frac{1}{2}\right)+H_{y}^{n}\left(i+\frac{1}{2}, j, k+\frac{1}{2}\right)-H_{y}^{n+1}\left(i+\frac{1}{2}, j, k-\frac{1}{2}\right)+H_{y}^{n}\left(i+\frac{1}{2}, j, k-\frac{1}{2}\right)}{2 d z}\right]
\end{aligned}
$$


Eq. (9) can be rewritten as follows:

$$
\begin{aligned}
& \frac{E_{x}^{n+1}-E_{x}^{n}}{d t}=\frac{1}{\varepsilon}\left[\frac{H_{z}^{n+1}(i, j+1, k)+H_{z}^{n}(i, j+1, k)-H_{z}^{n+1}(i, j, k)+H_{z}^{n}(i, j, k)}{2 d y}\right. \\
& \left.-\frac{\mathrm{H}_{y}^{n+1}(i, j, k+1)+H_{y}^{n+1}(i, j, k+1)-H_{y}^{n+1}(i, j, k)+H_{y}^{n+1}(i, j, k)}{2 d z}\right]
\end{aligned}
$$

Eq. (10) is deduced from twice central differences with combination of the $\mathrm{CN}$ theory.
Then, the numerical equation of the FDTD method of the 3D total space electromagnetic field based on the $\mathrm{CN}$ theory can be expressed as follows:

$$
\begin{aligned}
& \frac{E_{x}^{n+1}(i, j, k)-E_{x}^{n}(i, j, k)}{\Delta t}=\frac{\left(H_{z}^{n+1}(i, j+1, k)+H_{z}^{n}(i, j+1, k)\right)-\left(H_{z}^{n+1}(i, j, k)+H_{z}^{n}(i, j, k)\right)}{2 \varepsilon d y} \\
& -\frac{\left(H_{y}^{n+1}(i, j, k+1)+H_{y}^{n}(i, j, k+1)\right)-\left(H_{y}^{n+1}(i, j, k)+H_{y}^{n}(i, j, k)\right)}{2 \varepsilon d z} \\
& \frac{E_{y}^{n+1}(i, j, k)-E_{y}^{n}(i, j, k)}{\Delta t}=\frac{\left(H_{x}^{n+1}(i, j, k+1)+H_{x}^{n}(i, j, k+1)\right)-\left(H_{x}^{n+1}(i, j, k)+H_{x}^{n}(i, j, k)\right)}{2 \varepsilon d z} \\
& -\frac{\left(H_{z}^{n+1}(i+1, j, k)+H_{z}^{n}(i+1, j, k)\right)-\left(H_{z}^{n+1}(i, j, k)+H_{z}^{n}(i, j, k)\right)}{2 \varepsilon d x} \\
& \frac{E_{z}^{n+1}(i, j, k)-E_{z}^{n}(i, j, k)}{\Delta t}=\frac{\left(H_{y}^{n+1}(i+1, j, k)+H_{y}^{n}(i+1, j, k)\right)-\left(H_{y}^{n+1}(i, j, k)+H_{y}^{n}(i, j, k)\right)}{2 \varepsilon d x} \\
& -\frac{\left(H_{x}^{n+1}(i, j+1, k)+H_{x}^{n}(i, j+1, k)\right)-\left(H_{x}^{n+1}(i, j, k)+H_{x}^{n}(i, j, k)\right)}{2 \varepsilon d y} \\
& \frac{H_{x}^{n+1}(i, j, k)-H_{x}^{n}(i, j, k)}{\Delta t}=\frac{\left(E_{y}^{n+1}(i, j, k)+E_{y}^{n}(i, j+1, k)\right)-\left(E_{y}^{n+1}(i, j, k-1)+E_{y}^{n}(i, j, k-1)\right)}{2 \mu d z} \\
& -\frac{\left(E_{z}^{n+1}(i, j, k)+E_{z}^{n}(i, j, k)\right)-\left(E_{z}^{n+1}(i, j-1, k)+E_{z}^{n}(i, j-1, k)\right)}{2 \mu d y} \\
& \frac{H_{y}^{n+1}(i, j, k)-H_{y}^{n}(i, j, k)}{\Delta t}=\frac{\left(E_{z}^{n+1}(i, j, k)+E_{z}^{n}(i, j, k)\right)-\left(E_{z}^{n+1}(i-1, j, k)+E_{z}^{n}(i-1, j, k)\right)}{2 \mu d x} \\
& -\frac{\left(E_{x}^{n+1}(i, j, k)+E_{x}^{n}(i, j, k)\right)-\left(E_{x}^{n+1}(i-1, j, k)+E_{x}^{n}(i-1, j, k)\right)}{2 \mu d z} \\
& \frac{H_{z}^{n+1}(i, j, k)-H_{z}^{n}(i, j, k)}{\Delta t}=\frac{\left(E_{x}^{n+1}(i, j, k)+E_{x}^{n}(i, j, k)\right)-\left(E_{x}^{n+1}(i, j-1, k)+E_{x}^{n}(i, j-1, k)\right)}{2 \mu d y} \\
& -\frac{\left(E_{y}^{n+1}(i, j, k)+E_{y}^{n}(i, j, k)\right)-\left(E_{y}^{n+1}(i-1, j, k)+E_{y}^{n}(i-1, j, k)\right)}{2 \mu d x}
\end{aligned}
$$

where $\Delta t$ is the time step length. $d x, d y$, and $d z$ are the size of the spatial grids along the $\mathrm{x}-, \mathrm{y}-$, and z-axes. $i, j$, and $k$ are the number indexes of integer cells. Lastly, $n$ is the time step length [33-35].

\subsection{PML boundaries}

The space for the time-domain difference problem is also limited due to the limited calculation and memory space. Electromagnetic calculation in underground total space requires truncation by special boundary conditions. In the present study, the PML is selected. PML is a special medium 
with limited thickness, which covers the space for computer and creates a wave-matching condition on the basis of a virtual constitutive parameter. Such matching condition is unrelated with wave frequency and angle of incidence of boundary conditions.

The 3D PML matching condition is given as follows:

$$
\frac{\sigma_{\text {pex }}}{\varepsilon_{0}}=\frac{\sigma_{\text {pmx }}}{\mu_{0}}, \frac{\sigma_{\text {pey }}}{\varepsilon_{0}}=\frac{\sigma_{\text {pmy }}}{\mu_{0}}, \frac{\sigma_{\text {pez }}}{\varepsilon_{0}}=\frac{\sigma_{p m z}}{\mu_{0}}
$$

where $\sigma_{p e x}, \sigma_{p e y}, \sigma_{p m x}$, and $\sigma_{p m y}$ are specific and magnetic conductivities, which are introduced in the equation.

Compared with other boundary adsorption conditions, the PML boundary condition has the following advantages:

(1) When calculating the value of the PML boundary region, only the electric and magnetic field values of the boundary region have to be updated. However, the value of the entire grid region does not need to be updated.

(2) The boundary attenuation layer can be set flexible at the PML boundary. In other words, there are only forward and backward attenuations along the $\mathrm{x}$ direction, only left and right attenuations along the $y$ direction, and only upper and lower attenuations along the $\mathrm{z}$ direction. However, attenuations exist in the angular point region along the $x, y$, and $\mathrm{z}$ directions.

\subsection{Numerical stability conditions}

The FDTD algorithm must meet certain numerical stability conditions to calculate the electromagnetic properties of underground water-rich regions. Over time, the time step $(\Delta t)$ and space step lengths $(\Delta x, \Delta y, \Delta z)$ must meet Eq. (13). Among them, $\Delta \mathrm{t}$ is the time step length and $\Delta x, \Delta y, \Delta z$ are the minimum grid sizes along the $\mathrm{x}, \mathrm{y}$, and $\mathrm{z}$ directions, respectively.

$$
c \Delta t \leq \frac{1}{\sqrt{\frac{1}{(\Delta x)^{2}}+\frac{1}{(\Delta y)^{2}}+\frac{1}{(\Delta z)^{2}}}}
$$

This equation is the relation that the space and time discrete intervals must meet, that is, the Courant stability condition.

In the FDTD algorithm under 3D conditions $(\Delta x=\Delta y=\Delta z=\delta)$, Eq. (13) can be simplified as

$$
c \Delta t \leq \frac{\delta}{\sqrt{3}}
$$

The traditional FDTD algorithm solves the Maxwell differential equation by the classical explicit difference scheme, and its operation time step length is restricted by the Courant-Friedrichs-Lewy (CFL) stability condition. This implies that the selection of the time step length is determined by the size of the spatial dispersion grid. In addition, the space step length must be far shorter than the working wavelength. In particular, the space step length must be small enough to obtain the electromagnetic characteristics of fine structures accurately during simulation. Moreover, the time step length is suggested to be very small to ensure the stability of the solution, thus increasing the total computation time. However, this process cannot be realized at times. As an unconditional stability 3D FDTD algorithm, the CN-FDTD algorithm is based on the CN difference scheme, and each time step can be accomplished in one process. Numerical simulation results prove that the CN-FDTD algorithm can maintain good calculation accuracy when the time step length is significantly longer than that under conditional stability [36-38].

\section{Result Analysis and Discussion}

\subsection{Experimental design}

The heading face of the underground tunnel was constructed as a total space region. There's a fault water-rich zone in the unmined region in the directly ahead side. The water-bearing fault model is shown in Fig. 1.

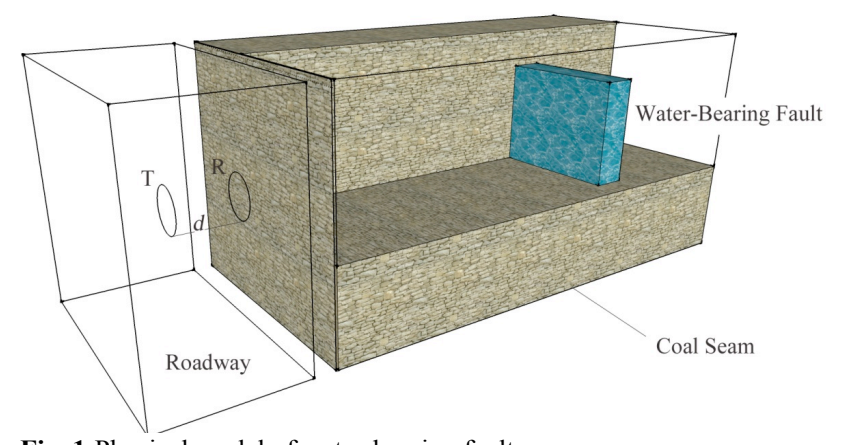

Fig. 1 Physical model of water-bearing fault

During simulation, all underground media except water are hypothesized as isotropic homogenous semi-infinite continuous space. The dielectric constants of air and underground media are 1 and 4, respectively. The dielectric constant of water is 81 , and its conductivity is 2 . The interface division is located at $35 \mathrm{~m}$ of the $\mathrm{y}$-axis, and the sources are arranged in a cyclic square with a side length of $1 \mathrm{~m}$. A total of 40 square sources are formed at an interval of $0.1 \mathrm{~m}$, which are located $5 \mathrm{~m}$ behind the interface and parallel to the XOZ coordinate plane. Similarly, observers are also arranged in cyclic squares with a side length of $2 \mathrm{~m}$. A total of 80 square observers are set at an interval of $0.1 \mathrm{~m}$, which are on the layer interface and parallel to the $\mathrm{XOZ}$ coordinate plane. The anomalous body is parallel to the $\mathrm{XOZ}$ coordinate plane. Water is the medium, and PML is used as the boundary condition.

\subsection{Analysis of simulation results}

4.2.1 Effects of excitation function on forward modeling To study the effects of excitation function on forward modeling, the dielectric constant, resistance value, and central frequency $(15 \mathrm{MHz})$ are fixed. In this case, the forward modeling results are only influenced by one variable (excitation function). The physical size of the total space is $100 * 150 * 80(\mathrm{~m})$ and the anomalous body is $70 \mathrm{~m}$ away from the interface. The gridding plot is set to $100 * 150 * 80$. The forward modeling results of different excitation function are shown in Fig. 2. 


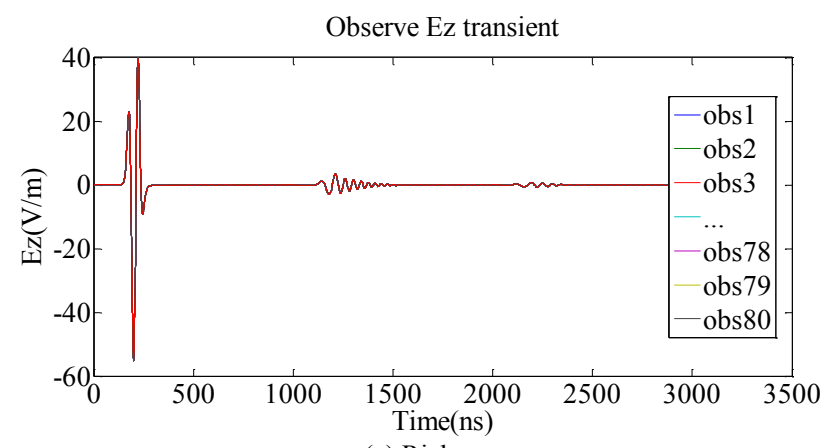

(a) Ricker

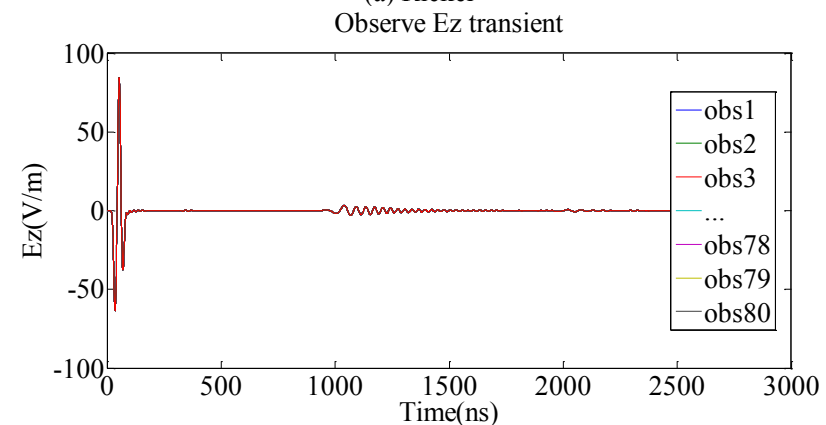

(b) BHW

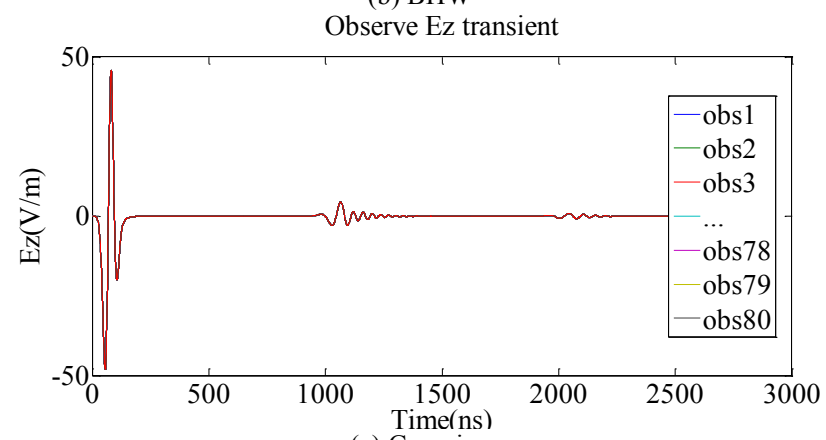

(c) Gaussian

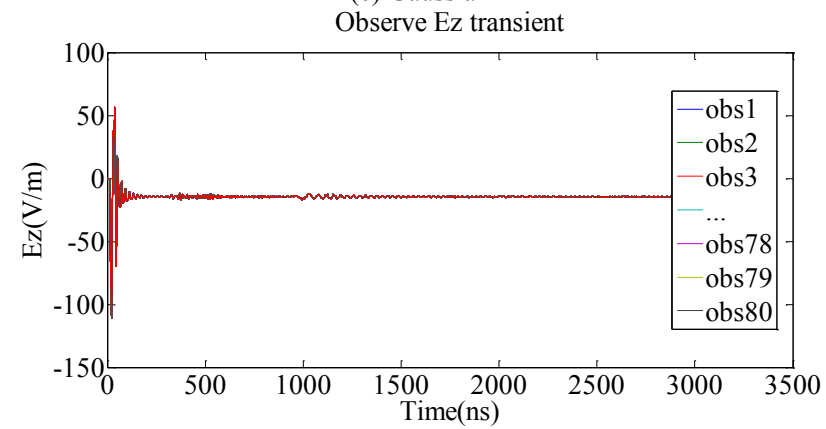

(d) Delta

Fig. 2 Forward modeling of different excitation functions

Response characteristic spectra of the anomalous body under four excitation functions are compared. Results reveal that the signal of the anomalous body can be detected by three excitation functions. However, delta forward modeling fails (Fig. 2(d)), and the accurate position of the anomalous body cannot be displayed by the waveform. The direct wave of BHW shows large amplitudes accompanied by a serious trailing phenomenon of the anomalous body. The Ricker and Gaussian response characteristic spectra have good effects as manifested by explicit direct and reflected waves of the anomalous body. The proportions of the reflected and direct waves of the anomalous body are $6.3 \%$ and $7.0 \%$, respectively. However, the amplitude of the Ricker direct wave is approximately $4.5 \%$ smaller than that of the Gaussian direct wave. In summary, Ricker is more applicable to be an excitation source.

\subsubsection{Effects of excitation source frequency on forward modeling}

To study the effects of central frequency on forward modeling, dielectric constant, resistance value, and excitation function are fixed. Therefore, the forward modeling results are only influenced by one variable, namely, central frequency. In this case, the physical size of the total space is $100 * 150 * 80(\mathrm{~m})$, and the anomalous body is $70 \mathrm{~m}$ away from the interface. The gridding plot is set to $100 * 150 * 80$. The forward modeling results under different central frequencies are shown in Fig. 3.

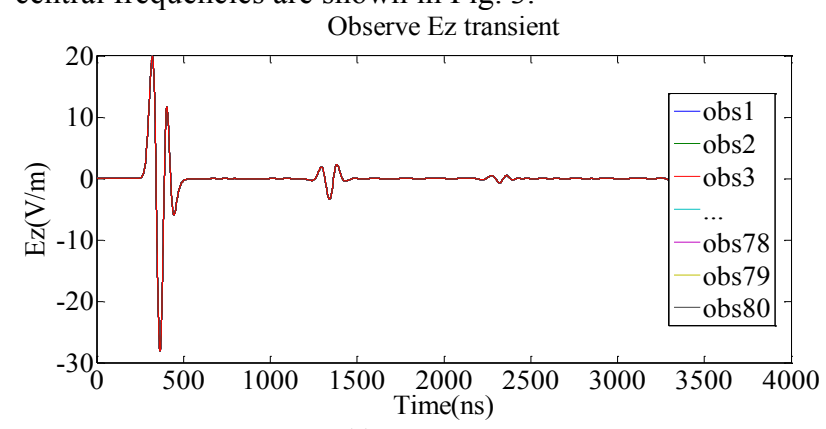

(a) $10.4 \mathrm{MHz}$

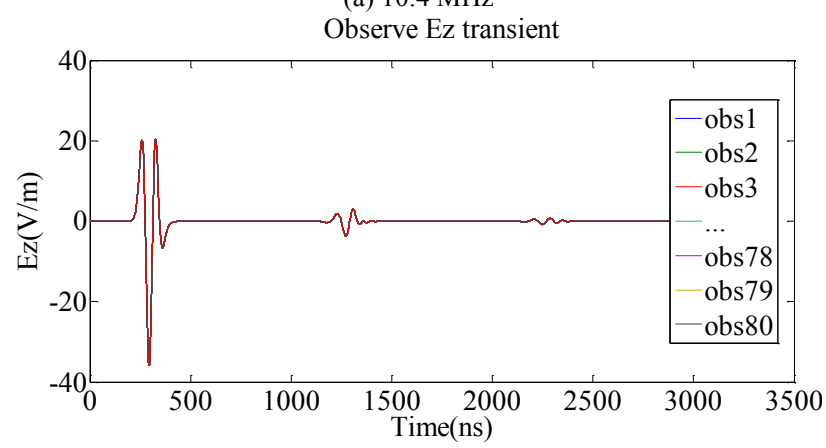

(b) $13 \mathrm{MHz}$

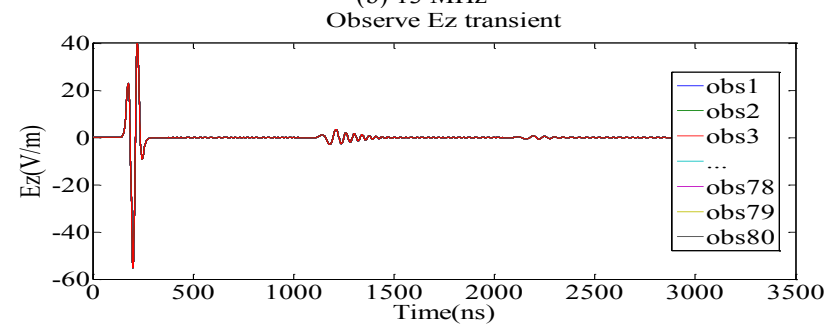

(c) $19.5 \mathrm{MHz}$

Observe Ez transient

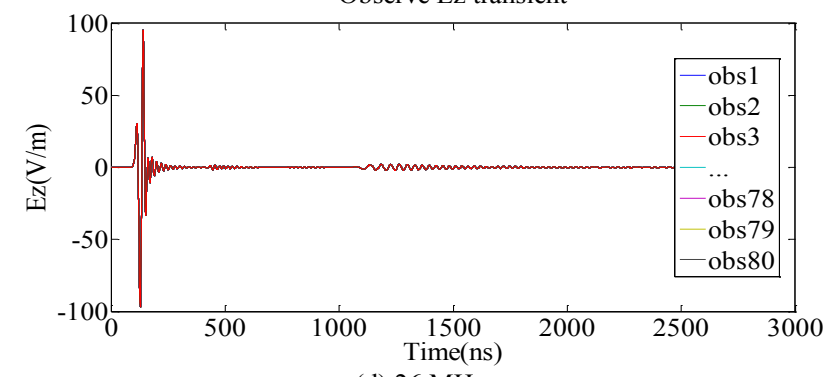

(d) $26 \mathrm{MHz}$

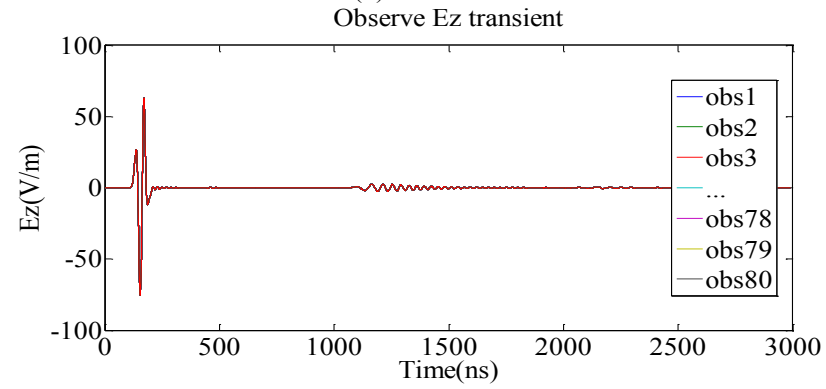


(e) $32.5 \mathrm{MHz}$

Observe Ez transient

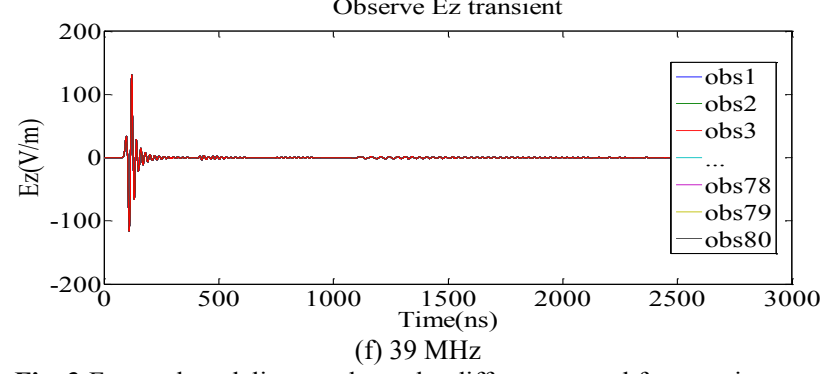

Fig. 3 Forward modeling results under different central frequencies

The response characteristic spectra of the anomalous body to different frequencies of the Ricker source are compared. All four frequencies of the Ricker source can detect signals from the anomalous body. With the increase of the central frequency of the Ricker source, the amplitude of the direct wave decreases first and then increases, accompanied by the trailing phenomenon. When the central frequency is increased to $32.5 \mathrm{MHz}$ and $39 \mathrm{MHz}$, the characteristic curve of the signal of the anomalous body becomes fuzzy, and the reflected wave of the anomalous body becomes extremely lengthy with low amplitude. Multiple waves occur in the simulation results (Fig. 3(e) and (f)). Multiple waves may seriously disturb the judgment of the anomalous body. With the decrease in frequency, the characteristic curve becomes increasingly clear. However, the forward modeling results are not negatively correlated with frequency. According to the analysis on forward modeling waveform under different Ricker central frequencies, the amplitudes of the reflected wave of the anomalous body at 10.4 and $26 \mathrm{MHz}$ (a serious interference of multiple waves occurs at frequencies of 32.5 and $39 \mathrm{MHz}$ are $3.4,3.7,3.5$, and $2.7 \mathrm{~V} / \mathrm{m}$. This finding reflects that the amplitude of the transmitting wave of the anomalous body increases first and then decreases with the increase in frequency. The time to detect the target anomalous body decreases with the increase in frequency, indicating the high propagation speed of electromagnetic waves in coals. According to previous studies, a high frequency may bring large dielectric loss, increased energy attenuation, and short detection distance.

\subsubsection{Effects of distance between anomalous body and interface on forward modeling}

To study the influence of distance between the anomalous body and interface on forward modeling, dielectric constant, resistance value, and excitation function are fixed. The forward modeling results are only affected by one variable (distance between the anomalous body and interface). In this case, the physical size of the total space is $100 * 300 * 80(\mathrm{~m})$. The central frequency of the Ricker source is set to $15 \mathrm{MHz}$, and the gridding plot is $100 * 300 * 80$. The forward modeling results under different distances between the anomalous body and interface are shown in Fig. 4.

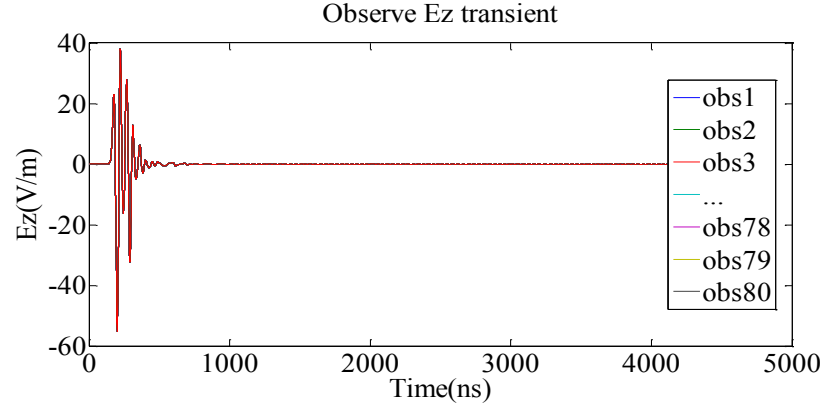

(a) $5 \mathrm{~m}$

Observe Ez transient

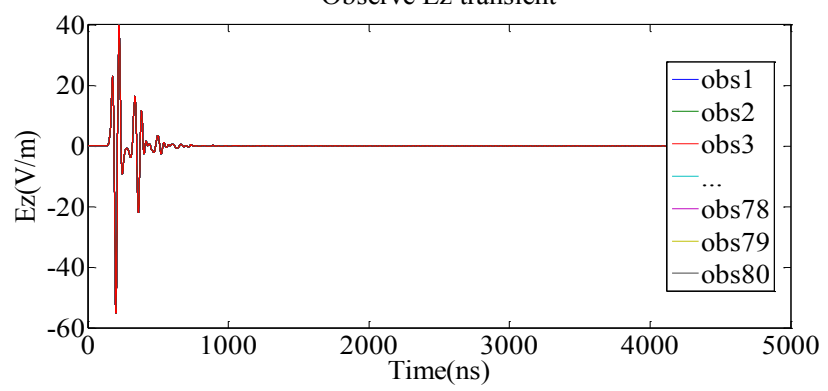

(b) $10 \mathrm{~m}$

Observe Ez transient

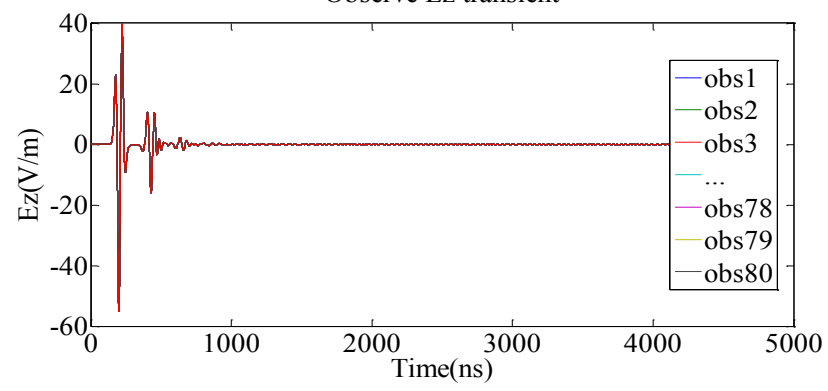

(c) $15 \mathrm{~m}$

Observe Ez transient

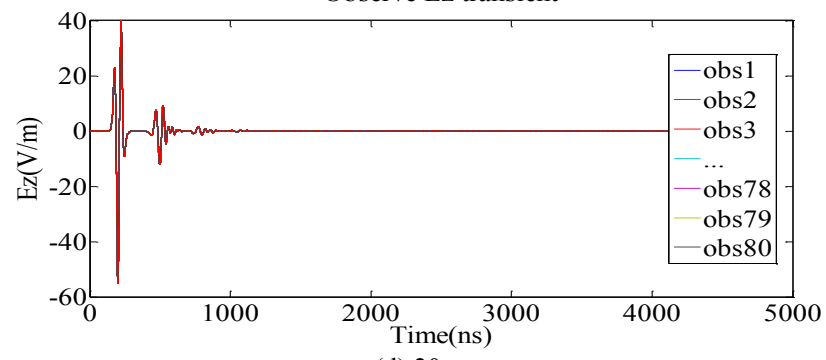

(d) $20 \mathrm{~m}$

Observe Ez transient

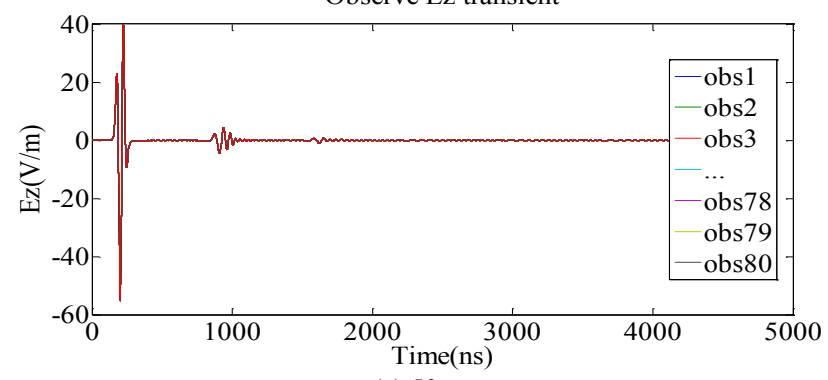

(e) $50 \mathrm{~m}$

Observe Ez transient

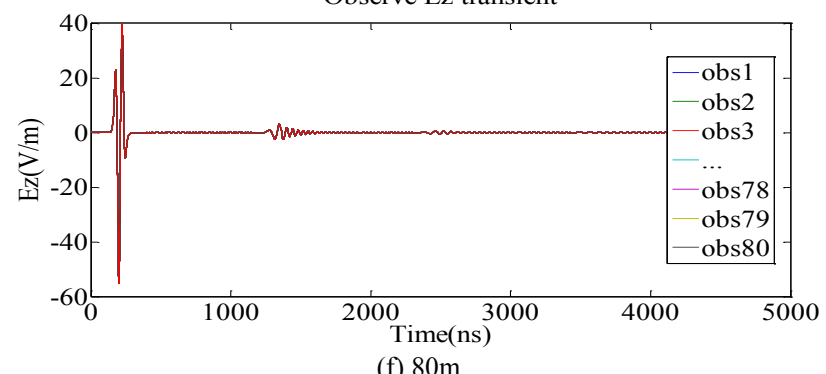

Observe Ez transient

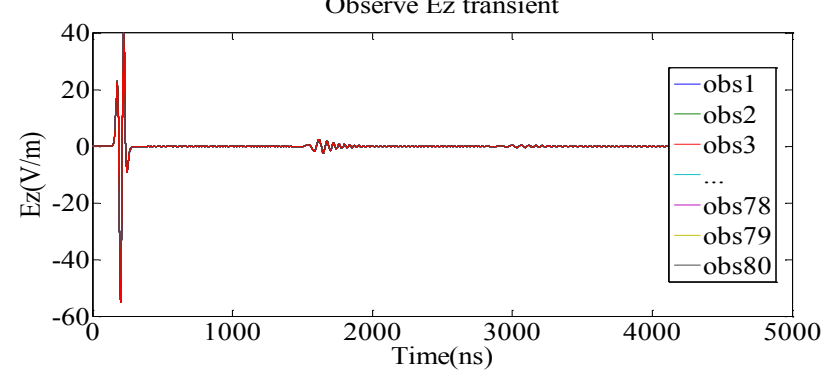


(g) $100 \mathrm{~m}$

Observe Ez transient

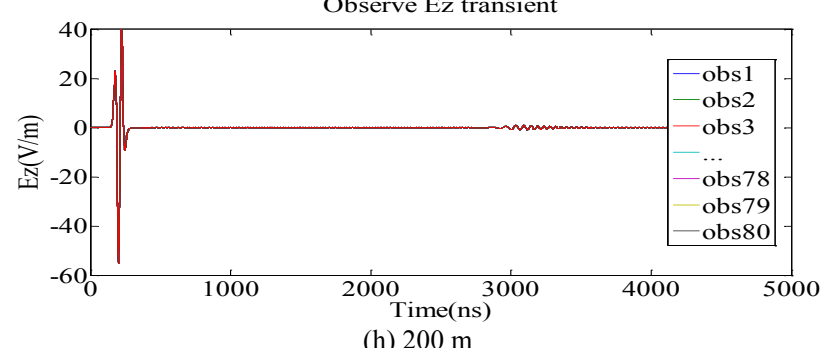

Fig. 4 Forward modeling under different distances between the anomalous body and the interface

The response characteristic spectra of the anomalous body to different distances between the anomalous body and interface are compared. The findings show that the signal of the anomalous body can be detected under all seven distances. With the increase in distance between the anomalous body and interface, the amplitude of the direct wave remains basically the same. The amplitudes of the reflected wave of the anomalous body when the distance ranged from $10 \mathrm{~m}$ to $200 \mathrm{~m}$ are $20,15,12,5,3,2.5$, and 2 $\mathrm{V} / \mathrm{m}$, which reflect the basically negative correlation between the intensity of the transmitting wave of the anomalous body and the distance between the anomalous body and the interface. As the distance between the anomalous body and the interface decreases, detecting the anomalous body in water-rich regions becomes easier. However, there is a certain blind area against detection, that is, the critical value of distance. In this simulation, the critical distance is $10 \mathrm{~m}$. The time to detect the target anomalous body increases with the increase in distance between the anomalous body and interface. The reflected wave of the anomalous body begins to develop the trailing phenomena when the distance is farther than $80 \mathrm{~m}$. However, it can still judge the position of the anomalous body. Therefore, the relative distance between the anomalous body and the interface can significantly influence the detection of the anomalous body.

According to the propagation law of the electromagnetic wave in lossy medium, the calculation formula of the propagation speed of the electromagnetic wave in coal can be gained:

$$
v=\frac{c}{\sqrt{\varepsilon_{r}}}
$$

where $v$ is the propagation speed of the electromagnetic wave in coal, $c$ is the speed of light, and $\varepsilon_{r}$ is the dielectric constant of coal.

Fig. 4 shows that the position of the anomalous body can be acquired by multiplying the propagation speed of the electromagnetic wave in coal by half time of the forward modeling wave (the propagation time of the electromagnetic wave is a round trip time; here, only the half time is needed). The positioning results of the anomalous body are shown in Table 1.

Table 1. The positioning results of the anomalous body

\begin{tabular}{|c|c|c|c|c|}
\hline \multirow[b]{2}{*}{ Model } & Analytical value (m) & True value (m) & \multirow[b]{2}{*}{ Absolute error (m) } & \multirow[b]{2}{*}{ Absolute error (m) } \\
\hline & $\begin{array}{l}\text { Distance between } \\
\text { anomalous body and } \\
\text { interface }\end{array}$ & $\begin{array}{l}\text { Distance between } \\
\text { anomalous body and } \\
\text { interface }\end{array}$ & & \\
\hline $\mathrm{c}$ & 15.075 & 15 & 0.075 & 0.50 \\
\hline $\mathrm{d}$ & 19.875 & 20 & 0.125 & 0.63 \\
\hline $\mathrm{e}$ & 49.500 & 50 & 0.500 & 1.00 \\
\hline $\mathrm{f}$ & 79.125 & 80 & 0.875 & 1.01 \\
\hline $\mathrm{g}$ & 99.375 & 100 & 0.625 & 0.63 \\
\hline $\mathrm{h}$ & 200.625 & 200 & 0.625 & 0.31 \\
\hline
\end{tabular}

\subsubsection{Effects of size of the anomalous body on forward modeling}

To study the influences of size of the anomalous body on forward modeling, dielectric constant, resistance value, excitation function, and central frequency are fixed. The forward modeling results are only affected by one variable (size of anomalous body). In this case, the physical size of the total space is $50 * 80 * 30(\mathrm{~m})$, the central frequency of the Ricker source is set to $15 \mathrm{MHz}$, and the anomalous body is $50 \mathrm{~m}$ away from the interface. The gridding plot is set to $50 * 80 * 30$. The forward modeling results under different sizes of the anomalous body are shown in Fig. 5.

The response characteristic spectra of the anomalous body to different sizes of the anomalous body are compared. The results show that the minimum physical size of the anomalous body that can be detected currently is $3 * 0.5 * 2$ (m), which indicates that $3 \mathrm{~m}^{3}$ of water causes no threats to the headwork. With the increase in the physical size of the anomalous body, the amplitude of the reflected wave increases accordingly. The amplitudes of the reflected wave are $0.42,0.64,0.66$, and $0.67 \mathrm{v} / \mathrm{m}$ when the physical size of the anomalous body ranges from $3 \mathrm{~m}^{3}$ to $8 \mathrm{~m}^{3}$. In other words, the intensity of the reflected wave of the anomalous body is positively related to the volume of the anomalous body. The current anomalous body is located $50 \mathrm{~m}$ behind the interface, and the water volume that can be detected is 3 $\mathrm{m}^{3}$.

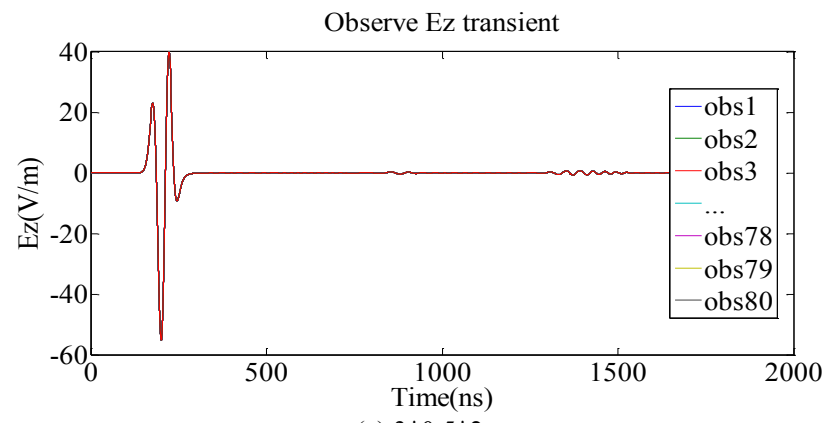

(a) $3 * 0.5 * 2$ 


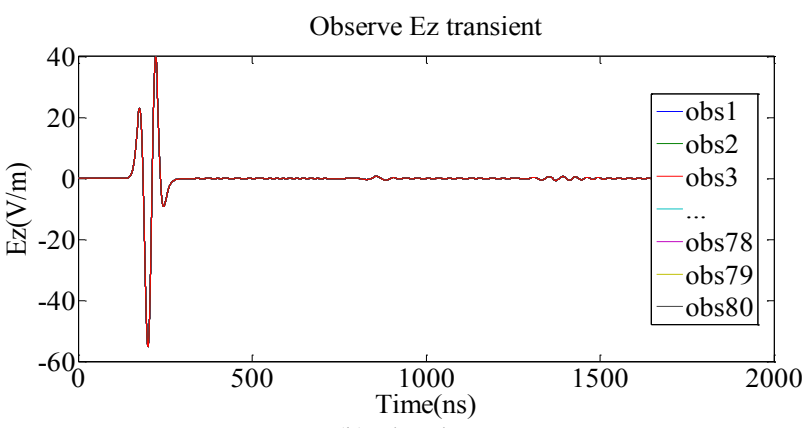

(b) $3 * 0.5 * 3$

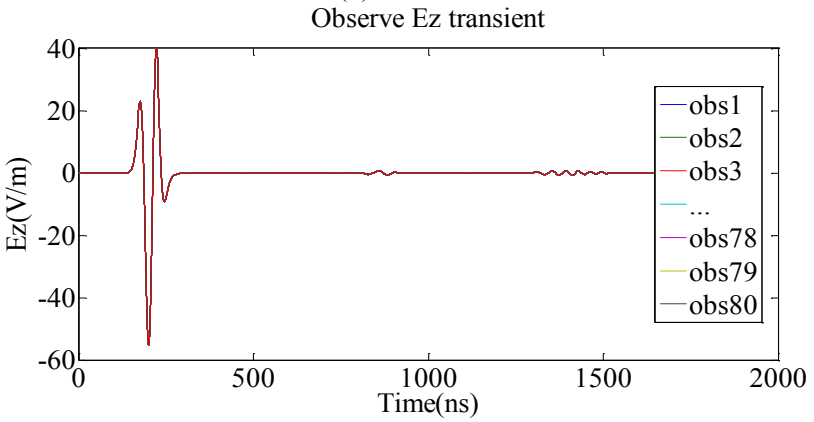

(c) $3 * 0.5 * 4$

Observe Ez transient

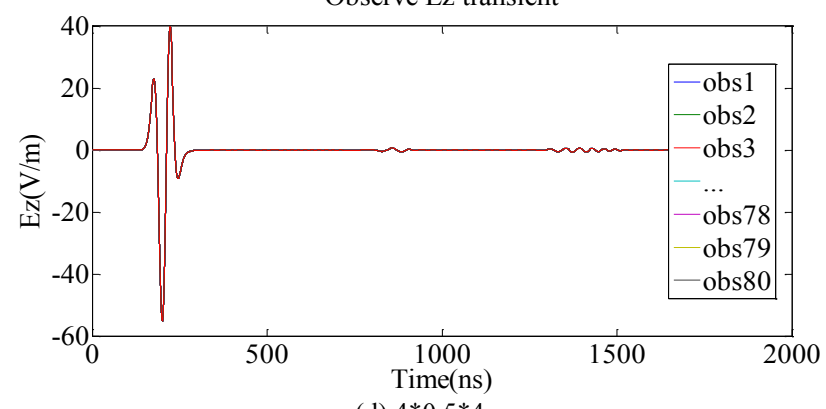

(d) $4 * 0.5 * 4$

Fig. 5 Forward modeling under different sizes of the anomalous body

\section{Conclusion}

In this study, the CN-FDTD algorithm was applied for the forward modeling of water-rich anomalous bodies in the underground mining environment. The validity of the $\mathrm{CN}$ FDTD algorithm in water-rich region detection in mines was proved. The algorithm overcame the constraint of stability condition on the traditional FDTD and increased the accuracy of water-rich region detection in mines. The following conclusions could be drawn:

(1) When the anomalous body is $50 \mathrm{~m}$ away from the interface (detection receiving point), the minimum size of the anomalous body that can be detected is $3 \mathrm{~m}^{3}$, and the body of water does not pose a threat to the safe production in mines. This result proves the applicability of the CN-FDTD algorithm for the electromagnetic detection of water-rich regions in mines.

(2) The CN-FDTD algorithm overcomes the constraint of the CFL stability condition on the step length selection in the traditional FDTD algorithm. The CN-FDTD algorithm achieves higher calculation speed and detection accuracy compared with the traditional FDTD algorithm.

(3) In the same detection model, the Ricker wavelet excitation source has a better detection effect given the same dielectric constant, resistance value, and central frequency.

(4) In CN-FDTD detection, the distance of the anomalous body has a certain range. When the distance to the receiving surface is smaller than $5 \mathrm{~m}$, there is a blind region against detection.

For the first time, the CN-FDTD algorithm is applied to the detection of underground anomalous bodies. However, the study remains a theoretical one. Future studies should develop a relevant transmitter-receiver set for mine survey. The CN-FDTD algorithm should be further optimized by combining the survey results and electromagnetic propagation characteristics in mines.

\section{Acknowledgements}

This work was supported by the Project of Science and Technology of Shaanxi (No.2018GY-151).

This is an Open Access article distributed under the terms of the Creative Commons Attribution License

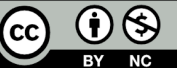

\section{References}

1. Zhang, D., Liu, Z., Yang, T., "Application of Transient Electromagnetic Method in Detection of Water-rich Areas in Coal Mines", Mineral Engineering Research, 28(04),2013, pp. 51-56.

2. Sun, H., Cheng, M., Wu, Q., "Three-Dimensional FDTD Forward Multi-Resolution Mesh Method for Transient Electromagnetics". Chinese Journal of geophysics, 61(12),2018,pp.5096-5104.

3. Xu, X. "Research on Transient Electromagnetic Field Response and Perspective Technology of Mine Water-bearing Structure". Doctoral Dissertation of China University of Mining and Technology, China, 2017, pp. 1-20.

4. Wang, C., Jiang, Q., "Application Analysis of Several Mine Electrical Methods". Coal geology in China, 29(3), 2017, pp. 7680.

5. Haber eldad W, Oldenburg-Douglas, R.-Shekhtman. "Inversion of time domain three-dimensional electromagnetic data". Geophysical Journal International, 171 (2), 2007, pp. 550-564.

6. Deng, X., "Inversion of Three-Dimensional Anomaly Characteristics of Transient Electromagnetism for Fixed Circuit Sources". Computing Techniques for Geophysical and Geophysical and Geochemical Exploration, 29(10), 2007, pp. 42-46.
7. Cheng, J., Li, M., Xiao, Y., "Study on Particle Swarm Optimization Inversion of Mine Transient Electromagnetic Method in Full Space". Journal of Geophysics, 57(10), 2014, pp. 3478-3484.

8. Li, X., Zhang, Y., Li, X., "Transient Electromagnetic Method Ground-Space Inverse Synthetic Aperture Imaging with Electrical Source" . China Journal of Geophysics, 58(1), 2015, pp. 277-288.

9. Xu, Z., "Three-dimensional forward modeling of magnetotelluric field in resistivity arbitrary anisotropic medium". Doctoral Dissertation of Ocean University of China, 2015, pp. 10-22.

10. Xing, X., Jiang, Q., Wu, Z., Li, W., Zhang, Q., "Study and Application of Full Space Fixed-Point Three-Dimensional Transient Electromagnetic Detection Technology”. Coal Geology \& Exploration, 46(S1), 2018, pp. 60-65.

11. Xu, H., Wang, D., Liu, Z., Deng, Z., Yu, J., Ding, Z., "Study on Simulation of ground-underground dual-source transient electromagnetic stereo detection". Geology and Exploration, 54(03) 2018, pp. 603-613.

12. Chang, J., "Three-dimensional numerical simulation and application of mine transient electromagnetic response in waterrich area of coal mine". Doctoral Dissertation of China University of Mining and Technology, 2017, pp. 2-30. 
13. Yu, J., "Exploration of Mine Transient Electromagnetic Method". Xuzhou: China University of Mining and Technology press, China, 2007.

14. Tian, Z., "Application of Comprehensive Mine Geophysical Prospecting in Water-Rich Detection of Coal Face Floor". Doctoral Dissertation of Xi'an University of Science and Technology, 2017, pp. 3-22.

15. Zhang, G., "Physical Modeling and Application of Transient Electromagnetic Method for Multicomponent Detection in Waterrich Area of Driving Face". Doctoral Dissertation of China University of Mining and Technology press, 2016, pp. 2-35.

16. Hou, W., Cao, S., Gao, X., "Evaluation of Water-rich Detection and Water Inflow of Roof Strata in the First Mining Face of Xin'an Coal Mine". China Minig Magazine, 25(S2), 2016, pp.270-272.

17. Zhuang, Y., "Study on Unconditionally Stable CN-FDTD Algorithm for Anisotropic Magnetic Plasma". Doctoral Dissertation of Tianjin Polytechnic University, 2018, pp. 13-30

18. Jiang, Z., Liu, S., Malekian, Reza, "Analysis of a Whole-Space Transient Electromagnetic Field in 2.5-Dimensional FDTD Geoelectric Modeling”. IEEE Access, 2017(5), pp. 18707-18714.

19. Yu, J., Reza Malekian, Chang J., Su, B., "Modeling of Whole-Space Transient Electromagnetic Responses Based on FDTD and its Application in the Mining Industry". IEEE Transactions on Industrial Informatics, 13(6), 2017, pp. 2974 - 2982.

20. Arne Van Londersele, Daniël De Zutter, Dries Vande Ginste, “An in-depth stability analysis of nonuniform FDTD combined with novel local implicitization techniques". Journal of Computational Physics, 342, 2017, pp. 177-193.

21. Jiang, H., "Study on CN-FDTD-PML Algorithm based on Auxiliary Differential Equation Method". Doctoral Dissertation of Tianjin Polytechnic University, 2015. pp. 10-28.

22. C.T. Schroder, W.R. Scott, G.D. Larson, "Elastic waves interacting with buried land mines: a study using the FDTD method". IEEE Transactions on Geoscience and Remote Sensing, 40(6), 2002, pp. $1405-1415$.

23. Fatih Kaburcuk, Veysel Demir, Atef Z. Elsherbeni, Ercument Arvas , Joseph R. Mautz, "Time-Domain Iterative Multiregion Technique for 3-D Scattering and Radiation Problems". IEEE Transactions on Antennas and Propagation, 64(5), 2016, pp. 1807 - 1817.

[24]Brad Martin, Atef Elsherbeni,Gregory E. Fasshauer, Mohammed Hadi, "Improved FDTD method around dielectric and PEC interfaces using RBF-FD techniques". In: Proceedings of 2018 International Applied Computational Electromagnetics Society Symposium. Denver, America, Institute of Electrical and Electronics Engineers Inc, 2018, pp. 1-2.

25. Mohammed F. Hadi , Atef Z. Elsherbeni, "Numerical dispersion and stability for three-dimensional cylindrical FDTD near the axis of rotation". In: Proceedings of 2017 11th European Conference on Antennas and Propagation, Paris, France, Institute of Electrical and Electronics Engineers Inc, 2017, pp. 936-938.
26. Babak Honarbakhsh, Shahrooz Asadi., "Analysis of Multiconductor Transmission Lines Using the CN-FDTD Method". IEEE Transactions on Electromagnetic Compatibility, 59(1), 2017, pp. $184-192$.

27. Shahrooz Asadi, Babak Honarbakhsh, "Linear Analysis of HighFrequency Field-Effect Transistors Using the CN-FDTD Method". IEEE Transactions on Microwave Theory and Techniques, 65(6), 2017, pp. $1946-1954$.

28. Adem G. Aydin, Atef Z. Elsherbeni, Bassem H. Henin, "Finite Difference Time Domain Formulations for 2D Scattering Problems With Oblique Incident Plane Waves", IEEE Transactions on Antennas and Propagation, 62(9), 2014, pp. 4820 - 4822.

29. Yang, Y., Han, Q., Chen, R., "The Three-Dimensional Unconditionally Stable FDTD algorithm based on Crank Nicolson method". IEEE Trans Mtt, 48(9), 2006, pp. 1550-1558.

30. Fu, Z., Shi, L., Huang, Z., Fu., S., "Study on Crank-Nicolson Unconditional Stability Method for Two-dimensional Acoustic Equation". Journal of Vibration and Shock, 36(17), 2017, pp. 7984.

31. Mohammed F. Hadi, Ravi C. Bollimuntha, Atef Z. Elsherbeni, Melinda Piket-May., "A Spherical FDTD Numerical Dispersion Relation Based on Elemental Spherical Wave Functions".IEEE Antennas and Wireless Propagation Letters, 17(5), 2018, pp. 784 788 .

32. Yang, J., "Continuous and Discontinuous Finite Element ThreeDimensional Numerical Simulation of the Earth's Electromagnetic Field". Doctoral Dissertation of University of Science and Technology of China, 2016, pp. 12-28.

33. Wang, W., Zhou, D., Liu, P., Tan, Y., "Step-by-step CN-FDTD analysis method for transmission lines terminated with arbitrary loads". Journal of Electronics and Information Technology, 35(01), 2013, pp. 209-214.

34. Zheng, K., Ge, D., Wei, B., "Crank-Nicolson FDTD Method for Analysis of Periodic Structure". Systems Engineering and Electronic Technology, 2005(08), 2005, pp.1332-1335.

35. Yang, L., Xu, H., Sun, D., Wang, H., “Z-FDTD Analysis of Electromagnetic Wave Propagation in Bianisotropic Dispersive Media”. The Chinese Journal of Radio Science, 30(03), 2015, pp. 423-428+448.

36. Li, J., Wu, P., "Efficient PML implementation based on the unconditionally stable CN-FDTD algorithm for anisotropic magnetized plasma". International Journal for Light and Electron Optics, 171(2018), 2018, pp. 468-475,

37. Wu, D., Chen, J., "Perfectly matched layer for Crank-Nicolson (CN) FDTD method". IEEE Antennas and Propagation Society Symposium, 2004(1), 2004, pp. 583-586

38. Song, D., Yang, H., Wang, G., "A research for plasma electromagnetic character using JEC-CN-FDTD algorithm based on ICCG method". International Journal for Light and Electron Optics, 127(3), 2016, pp. 1121-1125. 